\title{
An esophagectomy Surgical Apgar Score (eSAS)-based nomogram for predicting major morbidity in patients with esophageal carcinoma
}

\author{
Yong Xi", Weiyu Shen", Lijie Wang, Chaoqun Yu \\ Department of Thoracic Surgery, Ningbo Medical Center Lihuili Hospital, Ningbo 315040, China \\ Contributions: (I) Conception and design: Y Xi, C Yu ; (II) Administrative support: W Shen, C Yu; (III) Provision of study materials or patients: Y Xi, \\ W Shen, C Yu ; (IV) Collection and assembly of data: Y Xi, L Wang; (V) Data analysis and interpretation: Y Xi, C Yu ; (VI) Manuscript writing: All \\ authors; (VII) Final approval of manuscript: All authors. \\ "These authors contributed equally to this work. \\ Correspondence to: Chaoqun Yu, MD. 1111 Jiangnan Road, Department of Thoracic Surgery, Ningbo Medical Center Lihuili Hospital, Ningbo \\ 315000, China. Email: ycq2651@163.com.
}

\begin{abstract}
Background: Performing an esophagectomy for a malignancy presents an operation with an elevated risk of complications. The esophagectomy Surgical Apgar Score (eSAS) has been confirmed to be a strong predictor of major postoperative morbidity. The purpose of this study was to construct and establish an eSAS-based nomogram for predicting major morbidity after esophagectomy for esophageal carcinoma.

Methods: A total of 194 patients underwent radical esophagectomy for the malignant disease was analyzed by internal validation, and the clinical value was calculated on external validation $(n=135)$. The 30 -day major morbidity was recorded as the outcome. Univariable and multivariable logistic regression analysis analyzed the preoperative and intraoperative variables. An eSAS-based nomogram was constructed to predict the risk of major postoperative morbidity. The verification curves for the performance were drawn.

Results: Major morbidity occurred in 34.04\% ( $n=66$ ) of cases. Based on the final regression analysis, we proved that the eSAS had a highly linear association with major morbidity after esophagectomy. We further constructed a nomogram integrating the eSAS and clinical predictors [body mass index (BMI), American Society of Anesthesiologists (ASA) classification, and diabetes mellitus] to predict the probability of major postoperative morbidity. The performance of the eSAS-based nomogram was assessed and proven to be clinically useful by internal and external validation.

Conclusions: We constructed an eSAS-based nomogram that can effectively predict the risk of major morbidity after esophagectomy in patients with esophageal carcinoma. With a highly exact and exceedingly simple model, clinicians could more precisely ease the individual perioperative management for decreasing the postoperative complication.
\end{abstract}

Keywords: Esophagectomy; surgical Apgar score (SAS); major morbidity; nomogram; esophageal carcinoma

Submitted Dec 31, 2019. Accepted for publication Feb 18, 2020.

doi: $10.21037 /$ tcr.2020.02.56

View this article at: http://dx.doi.org/10.21037/tcr.2020.02.56

\section{Introduction}

Esophageal carcinoma is the seventh leading diagnosed malignancy worldwide, accounting for more than 450,000 new esophageal cancer cases (1). Esophagectomy with radical lymphadenectomy is a vital component of the treatment paradigm for such patients. Despite advances in treatment modalities and perioperative care, esophagectomy remains an operation with a relatively high risk of postoperative complications (2-4). In addition, various preoperative influences have been shown to affect 
Box 1 Esophagectomy Surgical Apgar Score (eSAS)

\begin{tabular}{lcccc}
\hline Intraoperative factors & 0 points & 1 point & 2 points & 3 points \\
\hline EBL $(\mathrm{mL})$ & $>450$ & $301-450$ & $191-300$ & $\leq 190$ \\
Lowest MAP $(\mathrm{mmHg})$ & $<40$ & $40-54$ & $55-69$ & $\geq 70$ \\
Lowest HR (beats/minute) & $>85$ & $76-85$ & $66-75$ & $56-65$ \\
\hline
\end{tabular}

EBL, estimated blood loss; MAP, mean arterial pressure; HR, heart rate.

short-term outcomes $(5,6)$. However, previous risk models have primarily focused on preoperative factors that might not be sufficient to predict patients with a high risk of morbidity $(6,7)$.

Although much attention has centered on preoperative risk factors, in general, it has been shown that rising preoperative factors influence short-term outcomes $(5,6)$. Gawande and colleagues (8) demonstrated that lowest heart rate, lowest mean arterial blood pressure, and estimated blood loss were all independent predictors of major morbidity in general surgical and vascular surgical patients, incorporating these measures into a 10 -point scoring system, referred to as the Surgical Apgar Score (SAS). Li and colleagues (9) completed a systematic review of eSAS to predict morbidity after esophagectomy and proved that eSAS is a valid assessment tool.

A predictive model for integrating intraoperative risk factors might be useful for helping clinicians improve treatment, to decrease complications $(10,11)$. Nomograms are often used as simple statistical predictive models that quantify the risk posed by a clinical event (12). Therefore, the purpose of this study was to construct and validate an esophagectomy Surgical Apgar Score (eSAS)based nomogram for predicting major morbidity after esophagectomy in patients with esophageal carcinoma.

\section{Methods}

\section{Patients}

From October 2015 to November 2018, a total of 214 consecutive patients who underwent esophagectomy in our department (Department of Thoracic Surgery, Ningbo Medical Center Lihuili Hospital, Ningbo, Zhejiang, China) were retrospectively reviewed. Patients were found from our institutional database of major thoracic surgical procedures. Patients who underwent no elective esophagectomy or surgery for benign disease were excluded $(n=20)$. This study was performed following the principles outlined in the Declaration of Helsinki and approved by the Ethical Committee and the Institutional Review Board. All patients supplied written informed consent before surgery and were contacted by telephone to obtain verbal informed consent.

\section{Preoperative and intraoperative data}

Preoperative characteristics of patients, including age, sex, body mass index (BMI), American Society of Anesthesiologists (ASA) classification, smoking history, abdominal or chest operation, comorbid disease (pulmonary disease, cardiovascular disease, diabetes mellitus, chronic renal or liver disease), and neoadjuvant chemotherapy or radiation therapy, were collected (13). Intraoperative data of patients, including surgical approaches, operative duration, and eSAS, were collected. Surgical techniques included Ivor Lewis esophagectomy and McKeown esophagectomy, performed through open esophagectomy (OE), hybrid (laparoscopy and thoracotomy or thoracoscopy and laparotomy), or minimally invasive esophagectomy (MIE) (laparoscopy and thoracoscopy). Regenbogen and colleagues had previously described the methodology for collecting and assigning points for the lowest heart rate and lowest mean arterial blood pressure (14). The ranges of estimated blood loss were modified from the original SAS scoring system and divided into four quartile values of estimated blood loss in our cohort. Details of the adjusted eSAS are shown in Box 1.

\section{Definition of outcomes}

Postoperative events were those defined by Esophagectomy Complications Consensus Group (ECCG) guidelines (15). The primary outcomes were postoperative 30 -day major morbidity based on STS GTSD risk models defined as the presence of one or more of the following postoperative adverse events: unexpected return to the operating room, anastomotic leak requiring medical or surgical treatment, reintubation, initial ventilatory support $>48$ hours, 
pneumonia, and recurrent nerve paresis $(16,17)$. Additional complications not meeting the STS GTSD risk model definitions for major complications were individually reviewed and evaluated according to the Clavien classification (18). Complications meeting the definition for Clavien class III complications (requiring surgical, endoscopic, or radiologic intervention) and class IV [requiring readmission to the intensive care unit (ICU) or considered life-threatening] were also categorized as major morbidity.

\section{Statistical analysis}

The purpose of this study was to construct a model for the prediction of major morbidity after esophagectomy for esophageal carcinoma. The associations between variables and the outcomes of interest were assessed with a test. Unadjusted odds ratios (ORs) were determined by univariable logistic regression analysis and reported with a $95 \%$ confidence interval (CI). Multivariate logistic regression analysis included variables with a $\mathrm{P}$ value of less than 0.1 by univariable analysis. A backward likelihood ratio selection procedure was then performed to determine the predictors in the final multivariable model.

Based on the final multivariable regression analysis, an eSAS-based nomogram for predicting the risk of major postoperative morbidity was developed. Respectively, through the receiver operating characteristic curve (AUC) and the calibration curve, discrimination and calibration were used to assess the performance of the nomogram (19). The value of the AUC ranged from 0.5 to 1.0, with 0.5 indicating a random chance and 1.0 indicating a perfect ability to discriminate the outcome with the model correctly (20). The calibration of the nomogram was assessed graphically by smoothing a scatter plot of the predicted probabilities and the actual probabilities. The clinical utility of the nomogram was determined by decision curve analysis (DCA) that was used to assess the potential of the clinical application of the nomogram by quantifying the net benefits.

Also, the nomogram was subjected to 1,000 bootstraps resamples for internal validation, and external validation was performed to assess their predictive accuracies using another set of 135 patients (Department of Cardiothoracic Surgery, Ningbo Medical Center Lihuili Hospital, Ningbo, Zhejiang, China). The cutoff scores with the best accuracy for major morbidity were used to group patients into lowrisk (below the cutoff) and high-risk (above the cutoff) nomogram score cohorts.

The standard chi-square test or Fisher's exact test was used for comparative analysis. The statistical analyses were performed using SAS 9.4 (SAS Institute Inc., Cary, NC, USA). Nomogram, ROC curves, and calibration curves were done with R 3.4.1 (The R Foundation for Statistical Computing, Vienna, Austria). For all analyses, the results of $\mathrm{P}<0.05$ were statistically significant.

\section{Results}

A total of 194 patients who underwent radical esophagectomy for malignant diseases at our institution were retrospectively reviewed. Ninety-two percent of the patients were male. The frequency of preoperative neoadjuvant chemoradiotherapy or chemotherapy was $24.74 \%(n=48)$. Surgical approaches included OE $(n=126$; $64.95 \%$ ), hybrid (24; $12.37 \%$ ), and MIE ( $\mathrm{n}=44 ; 22.68 \%$ ). Postoperative major morbidity occurred in $34.02 \%$ of patients $(n=66)$, while 30 -day mortality was zero in our cohort.

\section{Independent risk factors for major morbidity}

Results from the univariable analysis of the outcomes for major morbidity are shown in Table 1. After univariable analysis, the BMI, ASA classification, diabetes mellitus, operative duration, surgical approach, and eSAS were found to be significant risk factors. Independent variables with $\mathrm{P}<0.2$ derived by the univariable analysis were entered into the multivariable analysis. A backward likelihood ratio selection procedure was performed, and variables staying in the logistic regression model included BMI, ASA classification, diabetes mellitus, and eSAS. The multivariable analysis proved that BMI $<18.5 \mathrm{~kg} / \mathrm{m}^{2}$, ASA classification III-IV, diabetes mellitus, and eSAS were predictors of major morbidity (Table 2).

\section{Predictive nomogram for the probability of major morbidity}

Based on the multivariable regression analysis, a graphic nomogram was built that incorporated the four significant risk factors for the predicted probability of major postoperative morbidity (Figure 1). A total score was calculated using BMI, ASA classification, diabetes mellitus, and eSAS. The value of those factors was given a score on the point scale axis. A total score could be calculated 
Table 1 Patient characteristics and results of univariate analysis

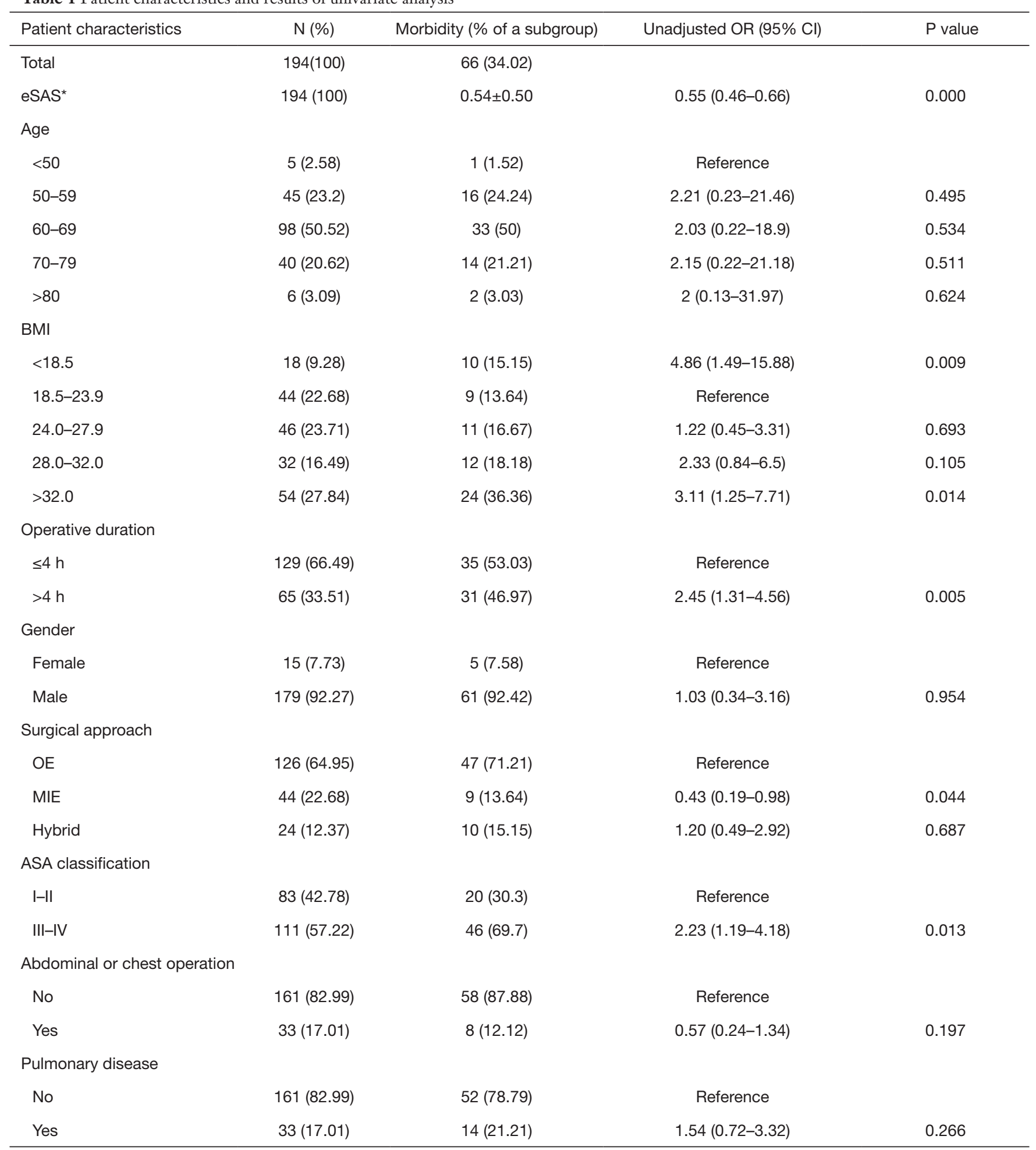

Table 1 (continued) 
Table 1 (continued)

\begin{tabular}{|c|c|c|c|c|}
\hline Patient characteristics & $\mathrm{N}(\%)$ & Morbidity (\% of a subgroup) & Unadjusted OR $(95 \% \mathrm{Cl})$ & $P$ value \\
\hline \multicolumn{5}{|l|}{ Cardiovascular disease } \\
\hline No & $108(55.67)$ & $40(60.61)$ & Reference & \\
\hline Yes & $86(44.33)$ & 26 (39.39) & $0.74(0.4-1.35)$ & 0.321 \\
\hline \multicolumn{5}{|l|}{ Diabetes mellitus } \\
\hline Yes & 38 (19.59) & 26 (39.39) & $6.28(2.9-13.61)$ & 0.000 \\
\hline \multicolumn{5}{|l|}{ Chronic renal disease } \\
\hline No & $172(88.66)$ & $61(92.42)$ & Reference & \\
\hline Yes & $22(11.34)$ & $5(7.58)$ & $0.54(0.19-1.52)$ & 0.241 \\
\hline Yes & $18(9.28)$ & $4(6.06)$ & $0.53(0.17-1.66)$ & 0.274 \\
\hline \multicolumn{5}{|l|}{ Smoking } \\
\hline No & $133(68.56)$ & $40(60.61)$ & Reference & \\
\hline Yes & $61(31.44)$ & 26 (39.39) & $1.73(0.92-3.24)$ & 0.088 \\
\hline \multicolumn{5}{|l|}{ Alcohol } \\
\hline No & $168(86.6)$ & 59 (89.39) & Reference & \\
\hline Yes & $26(13.4)$ & $7(10.61)$ & $0.68(0.27-1.71)$ & 0.414 \\
\hline \multicolumn{5}{|l|}{ Neoadjuvant CRT or CT } \\
\hline
\end{tabular}

${ }^{*}$, mean $\pm \mathrm{SD}$. Cl, confidence interval; OR, odds ratio.

Table 2 Variables included in the final multivariate model and adjusted odds ratios

\begin{tabular}{lcccc}
\hline Variable & Adjusted OR & $95 \% \mathrm{Cl}$ & $\mathrm{P}$ value \\
\hline eSAS & 0.49 & $0.39-0.62$ & \\
BMI & & & & \\
$<18.5$ & 6.03 & $1.44-25.24$ & 0.000 \\
$24.0-27.9$ & 0.68 & $0.19-2.44$ & 0.556 \\
$28.0-32.0$ & 0.75 & $0.19-2.98$ & 0.677 \\
$>32.0$ & 1.25 & $0.38-4.04$ & 0.714 \\
ASA classification III-IV & 2.83 & $1.2-6.71$ & 0.018 \\
Diabetes mellitus & 8.78 & $3.01-25.6$ & 0.000 \\
\hline
\end{tabular}

$\mathrm{Cl}$, confidence interval; OR, odds ratio. 


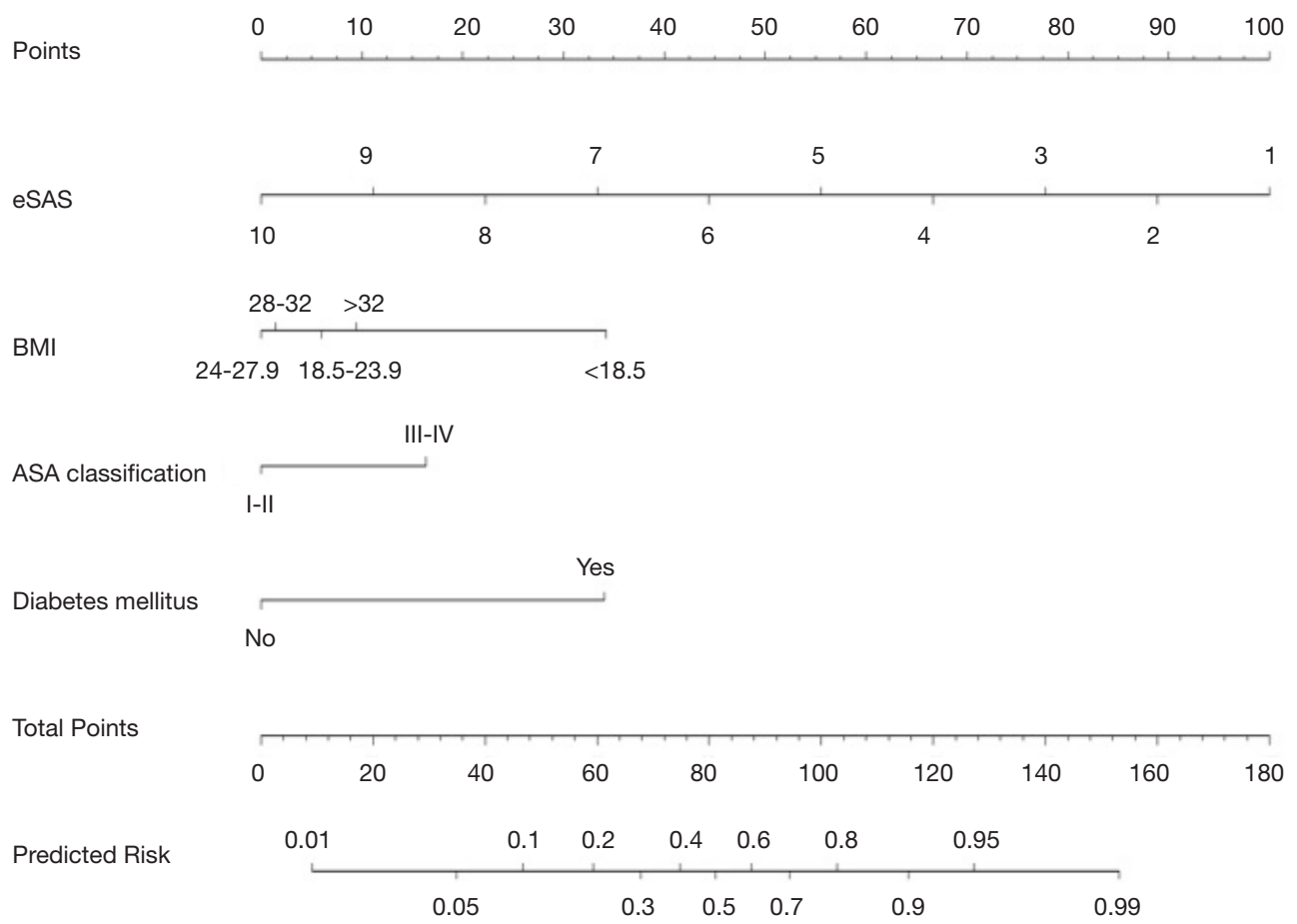

Figure 1 A nomogram integrating esophagectomy Surgical Apgar Score (eSAS) and preoperative risk factors associated with major postoperative morbidity.

Table 3 Major complication association with nomogram score

\begin{tabular}{|c|c|c|c|}
\hline Complication & Low-risk group $(n=117)$ & High-risk group ( $n=77$ ) & $P$ value \\
\hline Pneumonia & $4(3.4 \%)$ & $18(33.6 \%)$ & $<0.001$ \\
\hline Anastomotic or conduit leak* & $4(3.4 \%)$ & $13(16.9 \%)$ & 0.0012 \\
\hline Initial ventilatory support $>48$ hours & $1(0.9 \%)$ & $21(27.3 \%)$ & $<0.001$ \\
\hline Other Clavian class III or IV & $4(3.4 \%)$ & $19(24.7 \%)$ & $<0.001$ \\
\hline 30-day morbidity & $12(10.3 \%)$ & $54(70.1 \%)$ & $<0.001$ \\
\hline
\end{tabular}

Data are the numbers and percentages of patients. Some patients had more than 1 complication. *, requiring percutaneous intervention or reoperation (categorized as other Clavien Class III for analysis).

by adding every single score, and we could predict the probability of major postoperative morbidity.

\section{Postoperative outcomes}

Patients were divided into high- and low-risk groups by the score threshold of the nomogram, determined by the best accuracy (66.66) at this cutoff point concerning major morbidity. Complications suffered in five or more patients are shown in Table 3. Compared to the low-risk group, the high-risk group proved significantly more frequency of all major morbidities.

\section{Clinical utility of the nomogram}

The eSAS-based nomogram showed excellent discrimination, 

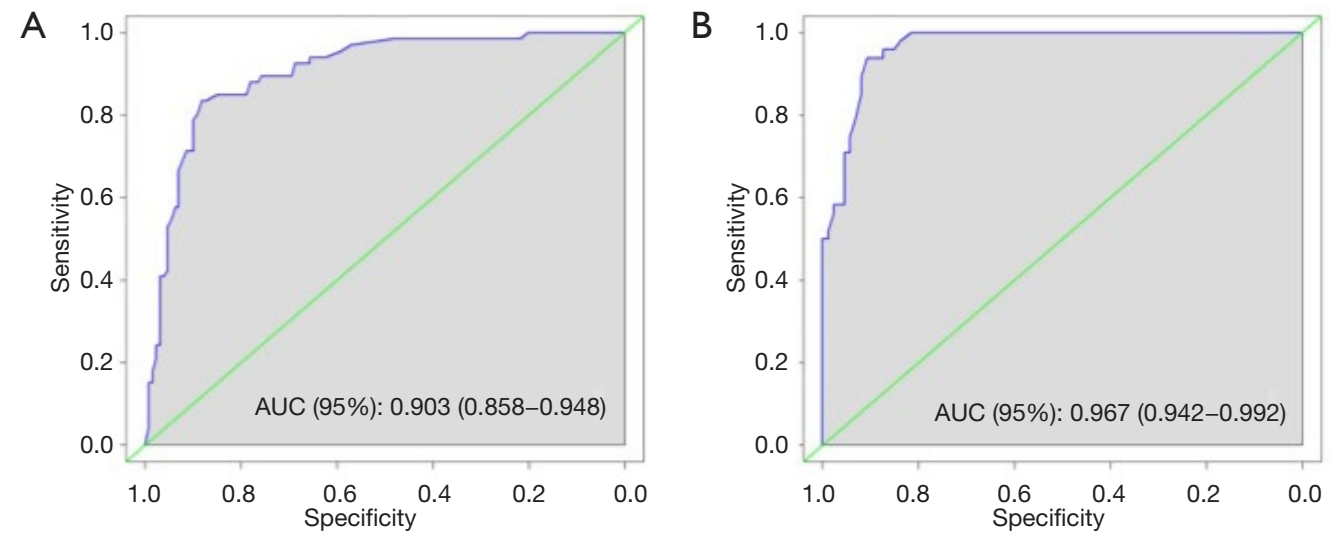

Figure 2 The receiver operating characteristic (ROC) curve for the nomogram. (A) Internal validation: the area under the ROC curve (AUC) $=0.903$ (95\% confidence interval: 0.858-0.948); (B) external validation: the AUC $=0.967$ (95\% confidence interval: $0.942-0.992$ ).

A

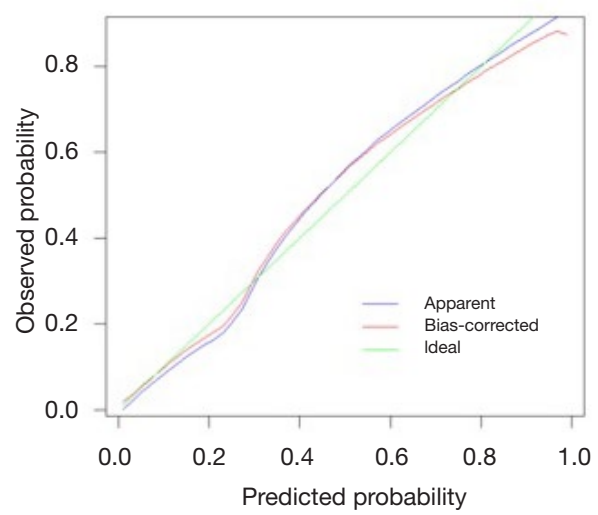

B

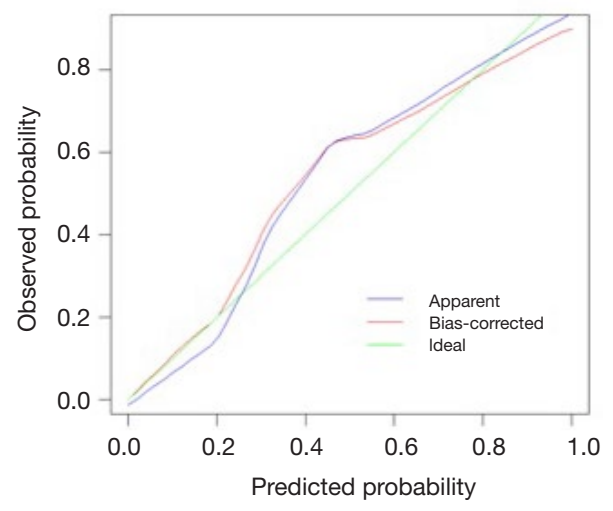

Figure 3 The calibration curve for the nomogram. The $\mathrm{x}$-axis is the eSAS-based nomogram-predicted probability, and the $y$-axis is the actual probability of major morbidity. (A) Internal validation; (B) external validation.

with an area under the receiver operating characteristics (ROC) curve of 0.903 (95\% CI: 0.858-0.948) for internal validation and 0.967 (95\% CI: 0.942-0.992) for external validation (Figure 2). Calibration curves are presented in Figure 3, with high consistency between the major morbidity probabilities predicted by the eSAS-based nomogram and the actual probability. The eSAS-based nomogram has promising potential for clinical application as better net benefits are ensured through the range of threshold probability for major complications compared with the treat-all or the treat-none option (Figure 4).

\section{Discussion}

Despite its many advantages, esophagectomy for esophageal carcinoma presently only is still a unique option for patients at elevated risk of postoperative morbidity. It is difficult to predict the risk of complications after esophagectomy for malignancy accurately. In recent studies, risk assessments incorporating preoperative risk factors into models to predict the probability of postoperative complications in esophageal carcinoma were used $(6-7,21)$. however, these reported risk models did not include intraoperative factors and concluded limited clinical applicability. To accurately predict the probability of major postoperative morbidity, In general, it has been shown that numerous preoperative factors influence short-term outcomes $(5,6)$.

The Apgar score was initially developed in 1953 by Virginia Apgar to predict newborn morbidity and mortality in obstetric and pediatric specialties (22). In 2007, Gawande and colleagues (8) modified this scoring system by evaluating 28 intraoperative variables and demonstrated that 

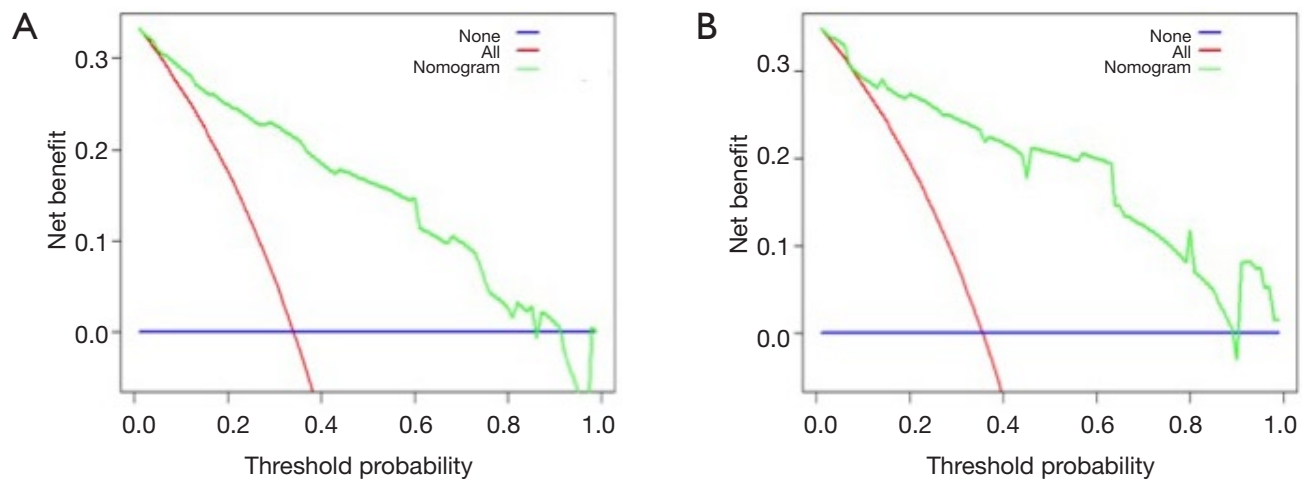

Figure 4 Decision curve analysis to evaluate the clinical utility of nomograms. The y-axis measures the net benefit. The green line is the nomogram. Using the eSAS-based nomogram to predict major postoperative morbidity could add more benefit than the treat-all-patients or the treat-none-patient strategy. (A) Internal validation; (B) external validation.

lowest heart rate, lowest mean arterial blood pressure, and estimated blood loss were independent predictors of major morbidity, referred to as the SAS. The SAS is a simple tool that may be well-suited for a complicated procedure like esophagectomy. Christopher and colleagues (13) were first to confirm the modified eSAS as being strongly associated with major morbidity after esophagectomy but did not have a linear association. Interestingly, we proved that eSAS had a significant linear association with major postoperative morbidity by univariable and multivariable analysis when analyzed as a continuous variable.

Nowadays, the SAS has been extensively proven to be an excellent predictor of major postoperative morbidity in patients with esophageal carcinoma $(13,23,24)$. Significant estimated blood loss, persistent tachycardia, and hypotension are strongly associated with adverse postoperative outcomes $(8,25,26)$. Lowest heart rate and mean arterial pressure are considered in not only physical backgrounds but also in anesthesia management decisions like depth of anesthesia, fluid infusion balance, and use of the circulating agent. A low eSAS score reflects inadequate perfusion and unstable intraoperative hemodynamics. The eSAS can reliably check the patient's hemodynamics during esophagectomy. Therefore, there are several advantages to using eSAS for patients with esophageal carcinoma.

A nomogram that includes preoperative factors has been developed and validated to calculate the probability of complications after esophagectomy $(27,28)$. To our knowledge, this is the first study that has constructed an eSAS-based nomogram to predict the risk of major postoperative morbidity for patients with esophageal carcinoma. In our nomogram, the eSAS is the most significant contributor to the probability of major morbidity after esophagectomy, followed by BMI and diabetes mellitus. The ASA classification showed the smallest effect on major postoperative morbidity.

Calibration plots revealed a good correlation between predicted and actual probability. The decision curve analysis showed a high potential clinical application of the eSASbased nomogram. This simple, intuitive graph could at once quantify the risk of major postoperative morbidity and allow for modification of perioperative care. Compared to the low-risk group, the high-risk group proved significantly more frequency of all major morbidity. In high-risk patients who underwent esophagectomy, postoperative ICU-level care could potentially decrease major morbidity (13). Therefore, it is recommended that high-risk patients be admitted to an ICU. Furthermore, proper risk-based triage also has the potential to reduce complications, resource utilization, and hospital costs by using riskmodified treatment strategies for high-risk patients. In our institution, one random prospective trial is ongoing to further prove the clinical effectiveness of the eSAS-based nomogram for patients with esophageal carcinoma.

Although the eSAS-based nomogram has been proven by internal and external validation to have good predictive accuracy for the probability of major morbidity after esophagectomy in esophageal carcinoma, our present study has several limitations. First, the definitions of major complications after esophagectomy remain heterogeneous and inconsistent, which could yield different outcomes $(15,29)$. Second, there was also inherent variability within 
the calculation of the estimated blood loss, as previous studies have noted $(13,30)$. Third, our nomogram was developed based on retrospective data, and so a large, randomized controlled, prospective study in a multicenter clinical trial could improve upon our model.

In conclusion, we constructed an eSAS-based nomogram that can effectively predict the risk of major morbidity after esophagectomy in patients with esophageal carcinoma. With a highly exact, exceedingly simple model, clinicians could more precisely ease the individual perioperative management for decreasing the postoperative complication.

\section{Acknowledgments}

Our abstract has been accepted for presentation at the 27th European Conference on General Thoracic Surgery, Dublin, Ireland, taking place between the 9th and 12th of June, 2019.

Funding: This work was supported by the major science and technology innovation in 2025 projects of Ningbo, China (Grant No. 2019B10039).

\section{Footnote}

Conflicts of Interest: All authors have completed the ICMJE uniform disclosure form (available at http://dx.doi. org/10.21037/tcr.2020.02.56). The authors have no conflicts of interest to declare.

Ethical Statement: The authors are accountable for all aspects of the work in ensuring that questions related to the accuracy or integrity of any part of the work are appropriately investigated and resolved. This study was performed following the principles outlined in the Declaration of Helsinki (as revised in 2013) and approved by the Ethical Committee and the Institutional Review Board (No. KY2019SL058-02). All patients supplied written informed consent before surgery and were contacted by telephone to obtain verbal informed consent.

Open Access Statement: This is an Open Access article distributed in accordance with the Creative Commons Attribution-NonCommercial-NoDerivs 4.0 International License (CC BY-NC-ND 4.0), which permits the noncommercial replication and distribution of the article with the strict proviso that no changes or edits are made and the original work is properly cited (including links to both the formal publication through the relevant DOI and the license).
See: https://creativecommons.org/licenses/by-nc-nd/4.0/.

\section{References}

1. Torre LA, Bray F, Siegel RL, et al. Global cancer statistics, 2012. CA Cancer J Clin 2015;65:87-108.

2. Zhang H, Yang Y, Shang Q, et al. Predictive value of preoperative weight loss on survival of elderly patients undergoing surgery for esophageal squamous cell carcinoma. Transl Cancer Res 2019;8:2752-8.

3. Kuo EY, Chang Y, Wright CD. Impact of hospital volume on clinical and economic outcomes for esophagectomy. Ann Thorac Surg 2001;72:1118-24.

4. Alghamedi A, Buduhan G, Tan L, et al. Quality of life assessment in esophagectomy patients. Ann Transl Med 2018;6:84.

5. Grotenhuis BA, Wijnhoven BP, Grüne F, et al. Preoperative risk assessment and prevention of complications in patients with esophageal cancer. J Surg Oncol 2010;101:270-8.

6. Zingg U, Langton C, Addison B, et al. Risk prediction scores for postoperative mortality after esophagectomy: validation of different models. J Gastrointest Surg 2009;13:611-8.

7. Warnell I, Chincholkar M, Eccles M. Predicting perioperative mortality after oesophagectomy: a systematic review of performance methods of multivariate models. $\mathrm{Br}$ J Anaesth 2015;114:32-43.

8. Gawande AA, Kwaan MR, Regenbogen SE, et al. An Apgar score for surgery. J Am Coll Surg 2007;204:201-8.

9. Li S, Zhou K, Li P, et al. Is surgical Apgar score an effective assessment tool for the prediction of postoperative complications in patients undergoing oesophagectomy? Interact Cardiovasc Thorac Surg 2018;27:686-91.

10. Lagarde SM, Reitsma JB, Maris AK, Preoperative prediction of the occurrence and severity of complications after esophagectomy for cancer with use of a nomogram. Ann Thorac Surg 2008;85:1938-45.

11. Nagy K, Murányi M, Nádas G, et al. Perioperative treatment after esophagogastric surgery. Magy Seb 2001;54:138-43.

12. Liang W, Zhang L, Jiang G, et al. Development and validation of a nomogram for predicting survival in patients with resected non-small-cell lung cancer. J Clin Oncol 2015;33:861-9.

13. Janowiak CF, Blasberg JD, Taylor L, et al. The Surgical Apgar Score in esophagectomy. J Thorac Cardiovasc Surg 2015;150: 806-12. 
14. Regenbogen SE, Ehrenfeld JM, Lipsitz SR, et al. The utility of the surgical Apgar score: validation in 4119 patients. Arch Surg 2009;144:30-6; discussion 37.

15. Low DE, Alderson D, Cecconello I, et al. International Consensus on Standardization of Data Collection for Complications Associated With Esophagectomy: Esophagectomy Complications Consensus Group (ECCG). Ann Surg 2015;262:286-94.

16. Raymond DP, Seder CW, Wright CD, et al. Predictors of Major Morbidity or Mortality After Resection for Esophageal Cancer: A Society of Thoracic Surgeons General Thoracic Surgeons General Thoracic Surgery Database Risk Adjustment Model. Ann Thorac Surg 2016;102:207-14.

17. Wright CD, Kucharczuk JC, O'Brien SM, et al. Predictors of major morbidity and mortality after esophagectomy for esophageal cancer: a Society of Thoracic Surgeons General Thoracic Surgery Database risk adjustment model.mJ Thorac Cardiovasc Surg 2009;137:587-95. Erratum in: J Thorac Cardiovasc Surg 2009;137:1581.

18. Dindo D, Demartines N, Clavien PA. Classification of surgical complications; a new proposal with evaluation in a cohort of 6336 patients and results of a survey. Ann Surg 2004;240:205-13.

19. Iasonos A, Schrag D, Raj GV, et al. How to build and interpret a nomogram for cancer prognosis. J Clin Oncol 2008;26: 1364-70.

20. Bandos AI, Rockette HE, Song T, et al. Area under the free response ROC curve (FROC) and a related summary index. Biometrics 2009;65:247-56.

21. Ferguson MK, Celauro AD, Prachand V. Assessment of a scoring system for predicting complications after esophagectomy. Dis Esophagus 2011;24:510-5.

Cite this article as: $\mathrm{Xi} \mathrm{Y,} \mathrm{Shen} \mathrm{W,} \mathrm{Wang} \mathrm{L,} \mathrm{Yu} \mathrm{C.} \mathrm{An}$ esophagectomy Surgical Apgar Score (eSAS)-based nomogram for predicting major morbidity in patients with esophageal carcinoma. Transl Cancer Res 2020;9(3):1732-1741. doi: 10.21037/tcr.2020.02.56
22. Apgar V. A proposal for a new method of evaluation of the newborn infant. originally published in July 1953, volume 32, pages 250-259. Anesth Analg 2015;120:1056-9.

23. Eto K, Yoshida N, Iwatsuki M, et al. Surgical Apgar Score predicted postoperative morbidity after esophagectomy for esophageal cancer. World J Surg 2016;40:1145-51.

24. Giugliano DN, Morgan A, Palazzo F, et al. Surgical Apgar score (SAS) predicts perioperative morbidity, mortality, and length of stay in patients undergoing esophagectomy at a high volume center. J Surg Oncol 2017;116:359-64.

25. Monk TG, Saini V, Weldon BC, et al. Anesthetic management and one-year mortality after noncardiac surgery. Anesth Analg 2005;100:4-10.

26. Zehetner J, DeMeester SR, Alicuben ET, et al. Intraoperative Assessment of Perfusion of the Gastric Graft and Correlation With Anastomotic Leaks After Esophagectomy. Ann Surg 2015;262:74-8.

27. Lagarde SM, Reitsma JB, Maris AK, et al. Preoperative prediction of the occurrence and severity of complications after esophagectomy for cancer with use of a nomogram. Ann Thorac Surg 2008;85:1938-45.

28. Grotenhuis BA, van Hagen P, Reitsma JB, et al. Validation of a nomogram predicting complications after esophagectomy for cancer. Ann Thorac Surg 2010;90:920-5.

29. Blencowe NS, Strong S, McNair AG, et al. Reporting of short-term clinical outcomes after esophagectomy; a systematic review. Ann Surg 2012;255:658-66.

30. Reynolds PQ, Sanders NW, Schildcrout JS, et al. Expansion of the surgical Apgar scores across all surgical subspecialties as a means to predict postoperative mortality. Anesthesiology 2011;114:1305-12. 